International Journal of Pure and Applied Mathematics

Volume 89 No. 3 2013, 393-400

ISSN: 1311-8080 (printed version); ISSN: 1314-3395 (on-line version)

url: http://www.ijpam.eu

doi: http://dx.doi.org/10.12732/ijpam.v89i3.10

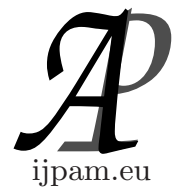

\title{
DISCRETENESS CRITERION FOR THE SPECTRUM OF THE SCHRÖDINGER OPERATOR ON WEIGHTED QUASIMODEL MANIFOLDS
}

\author{
Andrey Svetlov \\ Mathematics and IT Department \\ Volgograd State University \\ pr. Universitetskiy, 100, Volgograd, 400062, RUSSIA
}

\begin{abstract}
In this paper we study the spectrum of the Schrödinger operator on the weighted quasimodel manifolds. We obtain the discreteness criterion for this problem with some restrictions on the potential and geometry of the manifold.
\end{abstract}

AMS Subject Classification: $58 \mathrm{~J} 50$

Key Words: noncompact Riemannian manifold, quasimodel manifold, Schrödinger operator, discrete spectrum

\section{Introduction}

One of the contemporary directions in studying elliptic operators on noncompact Riemannian manifolds is the research of its spectrum. The first results appeared in the 70s of the twentieth century and concern the dependence of various spectral characteristics on the curvature of a manifold. For instance, for Laplace-Beltrami operator Yau [1] obtained lower bounds for the bottom of the spectrum on manifolds of negative Gaussian curvature. In the case when curvature is bounded from below by some nonpositive constant, an upper bound for the bottom of the spectrum was obtained by Cheng [2]. For two-dimensional surfaces of nonpositive Gaussian curvature, Pinsky [3] found two-sided estimates for the bottom of the laplacian spectrum and the bottom of the continuous spectrum in terms of the surface metric. Baider [4] obtained

Received: August 31, 2013

(c) 2013 Academic Publications, Ltd. url: www.acadpubl.eu 
a discreteness criterion for the spectrum of the Laplace-Beltrami operator on Riemannian warped products. Brooks [5] proved two-sided estimates for the bottom of the continuous spectrum in terms of the volume growth of a manifold.

For the Schrödinger operator Kondrat'ev and Shubin [6] obtained conditions of the spectrum discreteness for manifolds of bounded geometry and potentials bounded from below. In fact, they give a generalization of the Molchanovs criterion [8] for the spectrum to be discrete. Moreover, Z. Shen [7] found a criterion of the discreteness of the Schrödinger operator spectrum in terms of the potential growth, but it has some tough conditions on the manifold geometry.

In this article we consider the dependence of the spectrum of the Shrödinger operator on the metric of a quasimodel manifold. We describe these manifolds later in the paper. And let's note that the close results for the Laplacian spectrum were obtained by the author early [9].

\section{Schrödinger Operator on Warped Products}

We consider operators on Riemannian manifolds and start with the LaplaceBeltrami operator. Let $X$ be a smooth manifold of dimension $n$, assumed for simplicity connected. Moreover, suppose that a local coordinate system is given on $X$ and $\left\|g_{i j}\right\|$ is a Riemannian metric tensor on $X$; i.e., the metric has the form

$$
d s^{2}=\sum_{i, j=1}^{n} g_{i j} d x_{i} d x_{j} .
$$

Denote by $g^{i j}$ the entries of the matrix $\left\|g_{i j}\right\|^{-1}$ and put

$$
g=\operatorname{det}\left\|g_{i j}\right\|=\left(\operatorname{det}\left\|g^{i j}\right\|\right)^{-1} .
$$

Recall (see, for instance, [10]) that the divergence of a vector field $F=F_{i} \frac{\partial}{\partial x_{i}}$ on a manifold is

$$
\operatorname{div} F=\frac{1}{\sqrt{g}} \sum_{i=1}^{n} \frac{\partial}{\partial x_{i}}\left(\sqrt{g} F_{i}\right),
$$

and the components of the gradient of a function $f$ are given by

$$
(\nabla f)^{j}=\sum_{i=1}^{n} g^{i j} \frac{\partial f}{\partial x_{i}} .
$$

Assume some Borel measure $\mu$ is given on $X$, not necessarily Riemannian volume. We suppose that $\mu$ has density $\sigma(x)$, where $\sigma(x)$ is a smooth positive 
function. (Obviously, if $\mu$ is the Riemannian volume on $X$ then $\sigma(x) \equiv 1$.) The pair $(X, \mu)$ is called a weighted manifold. Then the divergence, defined as the dual of $\nabla$ with respect to the measure of the manifold, is calculated as follows:

$$
\operatorname{div}_{\mu} F=\frac{1}{\sigma(x) \sqrt{g}} \sum_{i=1}^{n} \frac{\partial}{\partial x_{i}}\left(\sigma(x) \sqrt{g} F_{i}\right) .
$$

It defines the Laplace-Beltrami operator

$$
-\Delta=-\operatorname{div}_{\mu} \nabla
$$

on $X$. It is considered on $L^{2}(X)$ with $C_{0}^{\infty}(X)$ domain which is dense in this space. Now, let $Z$ be a Riemannian manifold isometric to the product $X \times Y$ (where $X$ is an arbitrary manifold of dimension $n$ and $Y$ is a compact manifold of dimension $m$ ) with the metric

$$
d z^{2}=d x^{2}+\gamma^{2}(x) d y^{2},
$$

where $\gamma(x)$ is a smooth positive function and $d x^{2}$ and $d y^{2}$ are the metrics on $X$ and $Y$. Let $\mu$ be a Borel measure on $Z$ with density $\sigma(z)=\tau(x) \eta(y)$, and $\sigma(z)$ is a smooth positive function. Calculating the Laplace-Beltrami operator in curvilinear coordinates on the manifold, we arrive at the following

Lemma 1. The Laplace-Beltrami operator on $(Z, \mu)$ has the form

$$
-\Delta_{Z}=A_{0}+\gamma^{-2}\left(-\Delta_{\eta}\right),
$$

where $A_{0}$ is the Laplace-Beltrami operator on $X$ with weight $\gamma^{m}(x) \tau(x)$ and $-\Delta_{\eta}$ is the Laplace-Beltrami operator on $Y$ with the measure having density $\eta(y)$.

The proof of the Lemma can be found, for instance, in [11].

But also we consider the Schrödinger operator on $Z$ :

$$
-L_{Z}=-\Delta_{Z}+c(x) .
$$

Corollary 1. The Schrödinger operator on $(Z, \mu)$ has the form

$$
-L_{Z}=A_{0}+\gamma^{-2}\left(-\Delta_{\eta}\right)+c(x)=B+\gamma^{-2}\left(-\Delta_{\eta}\right),
$$

where $A_{0}$ and $B$ are the Laplace-Beltrami and Schrödinger operators on $X$ with weight $\gamma^{m}(x) \tau(x)$ and $-\Delta_{\eta}$ is the Laplace-Beltrami operator on $Y$ with the measure having density $\eta(y)$. 
The main subject of our research is the spectrum of the Schrödinger operator. We are interested in the two subsets of the spectrum of this operator: the set of all eigenvalues of finite multiplicity, the point spectrum, and the other part of the spectrum, the continuous spectrum. If the continuous spectrum is empty then we say that operator has discrete spectrum. In line with [12] and others, by the spectrum of a nonclosed but closable operator we mean the spectrum of the closure; i.e., the spectra of the Laplace-Beltrami and Schrödinger operators are in fact the spectra of the Friedrichs extensions of these operators (see, for instance, [12]).

Now, we state a discreteness criterion for the spectrum of the Schrödinger operator on $Z$. To this end, denote by $\nu$ the measure with weight $\gamma^{m}(x) \tau(x)$ on $X$.

Theorem 2. The Schrödinger operator on $(Z, \mu)$ has discrete spectrum if and only if the spectrum of the Schrödinger operator on $(X, \nu)$ is discrete.

The proof of this theorem can be found in [11], but the method we used for the proof is the same as in [4], where A. Baider proved the analogous theorem, but it's just for the Laplace-Beltrami operator and manifold $Z$ has no proper weight function.

\section{Discreteness of the Spectrum}

Now we consider a complete noncompact Riemannian manifold $M$ without boundary which is representable as $K \cup D$, where $K$ is a compact set and $D$ is isometric to the product $\mathbb{R}_{+} \times S_{1} \times S_{2} \times \cdots \times S_{k}$ (where $\mathbb{R}_{+}=(0,+\infty)$ and $S_{i}$ are compact Riemannian manifolds without boundary) with the metric

$$
d s^{2}=d r^{2}+q_{1}^{2}(r) d \theta_{1}^{2}+\cdots+q_{k}^{2}(r) d \theta_{k}^{2},
$$

where $d \theta_{i}^{2}$ is the metric on $S_{i}$ and $q_{i}(r)$ are smooth positive functions on $\mathbb{R}_{+}$. We assume $\operatorname{dim} S_{i}=n_{i}$ and so $\operatorname{dim} D=n_{1}+n_{2}+\cdots+n_{k}+1=n$. The behavior of harmonic functions (i.e., solutions to the equation $\Delta u=0$ ) on these manifolds was studied in detail by Losev [13], [14] who suggested to call the manifolds like $D$ simple warped products of order $k$. Clearly, these manifolds generalize the model manifolds, i.e., spherically symmetric ones. Consequently, particular instances of the manifolds under consideration $\left(k=1, q_{1}(r) \equiv q(r), S_{1}=\mathbb{S}^{n-1}\right.$ is the $(n-1)$-dimensional sphere) are $\mathbb{R}^{n}(q(r)=r)$, the hyperbolic space $\mathbb{H}^{n}$ $(q(r)=\sinh r)$, and all surfaces of revolution.

The manifold $M$ is a manifold with end. Since its end $D$ is a simple warped product, $D$ is the simplest case of a quasimodel manifold [13], [14]. We are 
interested in the case when $M$ is endowed with some Borel measure $\mu$ not necessarily coincident with Riemannian volume. In this case the pair $(M, \mu)$ is called a weighted manifold. Let this measure $\mu$ has the density

$$
\sigma\left(r, \theta_{1}, \ldots, \theta_{k}\right)=\tau(r) \eta_{1}\left(\theta_{1}\right) \ldots \eta_{k}\left(\theta_{k}\right) .
$$

Authors of the cited articles studied such properties of these manifolds as solvability of the Dirichlet problem, validity of Liouville-type theorems, etc. In this article we obtain a discreteness criterion for the spectrum of the Schrödinger operator on these manifolds.

Let's consider the Schrödinger operator on $M$ :

$$
-L=-\Delta+c(r),
$$

and denote

$$
F(r)=c(r)+\left(\frac{s^{\prime}(r)}{2 s(r)}\right)^{\prime}+\left(\frac{s^{\prime}(r)}{2 s(r)}\right)^{2},
$$

where $s(r)=\tau(r) q_{1}^{n_{1}}(r) \ldots q_{k}^{n_{\mathrm{k}}}(r)$.

Theorem 3. If $F(r)>-K(K=$ const $)$, the spectrum of the Schrödinger operator $-L$ on the manifold $(M, \mu)$ is discrete if and only if

$$
\forall \omega>0 \quad \lim _{r \rightarrow \infty} \int_{r}^{r+\omega} F(r) d r=+\infty .
$$

Proof. It follows from the decomposition principle $[15,16]$ that the spectrum of the Schrödinger operator on a manifold is discrete if and only if it is discrete outside every compact set of the manifold; i.e., discreteness of the spectrum on $M=K \cup D$ is equivalent to that on $D$. Take $X=\mathbb{R}_{+} \times S_{1} \times S_{2} \times \cdots \times S_{k-1}$ and $Y=S_{k}$ and consider $Z=X \times Y$ with the metric

$$
d z^{2}=d x^{2}+q_{k}^{2}(r) d y^{2}
$$

and the Borel measure $\tau_{1}(x) \eta_{k}\left(\theta_{k}\right)$, where

$$
\tau_{1}(x)=\tau(r) \eta_{1}\left(\theta_{1}\right) \ldots \eta_{k-1}\left(\theta_{k-1}\right) ;
$$

i.e., $Z$ coincides with $D$. By Theorem 2, the Schrödinger operator on $D$ has discrete spectrum if and only if so is the spectrum of the Schrödinger operator on $X$ with weight

$$
q_{k}^{n_{\mathrm{k}}}(r) \tau_{1}(x)=q_{k}^{n_{\mathrm{k}}}(r) \tau(r) \eta_{1}\left(\theta_{1}\right) \ldots \eta_{k-1}\left(\theta_{k-1}\right) .
$$


Take $X_{1}=\mathbb{R}_{+} \times S_{1} \times S_{2} \times \cdots \times S_{k-2}$ and $Y_{1}=S_{k-1}$ and consider $Z_{1}=X_{1} \times Y_{1}$ with the metric

$$
d z_{1}^{2}=d x_{1}^{2}+q_{k-1}^{2}(r) d y_{1}^{2}
$$

and weight $q_{k}^{n_{\mathrm{k}}}(r) \tau_{2}\left(x_{1}\right) \eta_{k-1}\left(\theta_{k-1}\right), \tau_{2}\left(x_{1}\right)=\tau(r) \eta_{1}\left(\theta_{1}\right) \ldots \eta_{k-2}\left(\theta_{k-2}\right)$; i.e., the Schrödinger operator on $Z_{1}$ is the operator whose discreteness is inspected. Applying Theorem 2 once again, we come to the following: discreteness of the spectrum of the $-L$ on $X$ with the above weight is equivalent to that of the Schrödinger operator on $X_{1}$ with the weight

$$
q_{k-1}^{n_{\mathrm{k}-1}}(r) q_{k}^{n_{\mathrm{k}}}(r) \tau_{2}\left(x_{1}\right)=q_{k-1}^{n_{\mathrm{k}-1}}(r) q_{k}^{n_{\mathrm{k}}}(r) \tau(r) \eta_{1}\left(\theta_{1}\right) \ldots \eta_{k-2}\left(\theta_{k-2}\right)
$$

Repeating the argument $k-2$ times, we finally conclude that discreteness of the spectrum of the Schrödinger operator on $D$ is equivalent to that of the Sturm-Liouville operator on $\mathbb{R}_{+}$with the weight $\tau(r) q_{1}^{n_{1}}(r) \ldots q_{k}^{n_{\mathrm{k}}}(r)$.

Put $s(r)=\tau(r) q_{1}^{n_{1}}(r) \ldots q_{k}^{n_{\mathrm{k}}}(r)$. Then the operator has the form

$$
B=s^{-1}(r) \frac{d}{d r}\left(s(r) \frac{d}{d r}\right)+c(r) .
$$

Removing parentheses we get:

$$
B u=-u^{\prime \prime}(r)-\frac{s^{\prime}(r)}{s(r)} u^{\prime}(r)+c(r) u(r) .
$$

Denote bu $\zeta(r)=s^{\frac{1}{2}}(r) u(r)$, i.e. $u=s^{-\frac{1}{2}}(r) \zeta(r)$. Then:

$$
\begin{gathered}
u^{\prime}=\zeta^{\prime} s^{-\frac{1}{2}}-\frac{1}{2} s^{-\frac{3}{2}} s^{\prime} \zeta \\
u^{\prime \prime}=\zeta^{\prime \prime} s^{-\frac{1}{2}}-\frac{1}{2} s^{-\frac{3}{2}} s^{\prime} \zeta^{\prime}-\frac{1}{2} s^{-\frac{3}{2}} s^{\prime} \zeta^{\prime}+\frac{3}{4} s^{-\frac{5}{2}} s^{2} \zeta-\frac{1}{2} s^{-\frac{3}{2}} s^{\prime \prime} \zeta .
\end{gathered}
$$

So we get:

$$
\begin{gathered}
B u=-\zeta^{\prime \prime} s^{-\frac{1}{2}}+\zeta^{\prime} s^{-\frac{3}{2}} s^{\prime}-\frac{3}{4} \zeta s^{-\frac{5}{2}} s^{2}+\frac{1}{2} \zeta s^{-\frac{3}{2}} s^{\prime \prime}-\zeta^{\prime} s^{-\frac{3}{2}} s^{\prime}+\frac{1}{2} \zeta s^{-\frac{5}{2}} s^{2}+\zeta c s^{-\frac{1}{2}} \\
B_{0} \zeta=s^{\frac{1}{2}} B u=-\zeta^{\prime \prime}-\zeta\left(\frac{s^{\prime 2}}{4 s^{2}}-\frac{s^{\prime \prime}}{2 s}-c\right) .
\end{gathered}
$$

To this end, denote by

$$
F(r)=c(r)+\frac{s^{\prime \prime}}{2 s}-\frac{s^{\prime 2}}{4 s^{2}}=c(r)+\left(\frac{s^{\prime}(r)}{2 s(r)}\right)^{\prime}+\left(\frac{s^{\prime}(r)}{2 s(r)}\right)^{2}
$$


thus we get operator:

$$
B_{0}=-\frac{d^{2}}{d r^{2}}+F(r)
$$

Now, we can apply Molchanov's discreteness criterion [8]: if function $F(r)$ is bounded from below, then the spectrum of the operator $B_{0}$ and, simultaneously, the Schrödinger operator $-L$ on the manifold $M$, are discrete if and only if mean value of function $F(r)$ tends to $+\infty$ when $r \rightarrow \infty$ :

$$
\forall \omega>0 \quad \lim _{r \rightarrow \infty} \int_{r}^{r+\omega} F(r) d r=+\infty .
$$

Theorem is proved.

Remark. As follows easily from the proof of the last theorem, a similar criterion is also valid for quasimodel manifolds $M=K \cup D_{1} \cup D_{2} \cup \cdots \cup D_{p}$ , where $K$ is a compact and $D_{i}$ are simple warped products. In this case the condition of the theorem should hold for each end $D_{i}$.

\section{Acknowledgments}

The reported study was partially supported by RFBR, research project No. 13-01-97038-r_povolzhye_a.

\section{References}

[1] S.T. Yau, Isoperimetric constants and the first eigenvalue of a complete Riemannian manifold, Ann.Sci.Ecole Norm.Sup., 8, No. 4 (1975), 487-507.

[2] S.Y. Cheng, Eigenvalue comparison theorem and its geometric applications, Math.Z., 143 (1975), 289-297, doi: 10.1007/BF01214381.

[3] M. Pinsky, The spectrum of the Laplacian on a manifold of negative curvature I, J.Diff.Geom., 13 (1978), 87-91.

[4] A. Baider, Noncompact Riemannian manifolds with discrete spectra, J.Diff.Geom., 14 (1979), 41-57.

[5] R. Brooks, A relation between growth and the spectrum of the Laplacian, Math. Z., 178 (1981), 501-508, doi: 10.1007/BF01174771. 
[6] V. Kondratev, M. Subin, Discreteness of spectrum for the Schrödinger operators on manifolds of bounded geometry, Operator theory: Advances and Applications, 110 (1999), 185-226.

[7] Z. Shen, The spectrum of Schrödinger operators with positive potentials in Riemannian manifolds, Proc. of Amer.Math.Soc., 131, No. 11 (2003), 3447-3456, doi: 10.1090/S0002-9939-03-06968-5.

[8] A.M. Molchanov, On conditions for discreteness of the spectrum of selfadjoint differential equations of the second order, Tr. Mosk. Mat. Obs., 2 (1953), 169-199.

[9] A.V. Svetlov, A discreteness criterion for the spectrum of the LaplaceBeltrami operator on quasimodel manifolds, Siberian Mathematical Journal, 43, No. 6 (2002), 1103-1111, doi: 10.1023/A:1021129703899.

[10] A.A. Grigor'yan, Analytic and geometric background of recurrence and non-explosion of the Brownian motion on Riemannian manifolds, Bull. Amer. Math. Soc., 36 (1999), 135-249, doi: 10.1090/S0273-0979-99-007764.

[11] A.V. Svetlov, The spectrum of the Schrodinger operator on the warped products, Vestnik VolGU [in Russian], 7 (2002), 12-19.

[12] M.C. Reed, B. Simon (Eds.), Methods of Modern Mathematical Physics: Functional analysis. 1 (Vol. 1), Academic Press, London (1980).

[13] A.G. Losev, On some Liouville theorems on noncompact Riemannian manifolds, Siberian Math. J., 39, No. 1 (1998), 74-80, doi: $10.1007 / \mathrm{BF} 02732362$.

[14] A.G. Losev, E.A. Mazepa, Bounded solutions of the Schrödinger equation on Riemannian products, St. Petersburg Math. J., 13, No. 1 (2001), 57-73.

[15] I. M. Glazman, Direct Methods of Qualitative Spectral Analysis of Singular Differential Operators, Israel Program for Scientific Translations, Jerusalem, 1965.

[16] M. Schechter, Spectra of partial differential operators, North-Holland, Amsterdam (1971). 